\title{
Two Combinatorial Problems in the Plane
}

\author{
P. Erdös ${ }^{1}$ and G. Purdy ${ }^{2}$
}

${ }^{1}$ Mathematical Institute, Hungarian Academy of Sciences, PO Box 127, H-1364, Budapest, Hungary

${ }^{2}$ Department of Computer Science, University of Cincinnati,

Cincinnati, OH 45221-0008, USA

Abstract. The authors discuss two problems involving $n$ points in the plane and the $t$ lines they form.

\section{The First Problem}

Let $n$ points be given in the plane, not all lying on a line. Let $t_{k}$ denote the number of lines containing exactly $k$ of the points, and let $t=t_{2}+t_{3}+t_{4}+\cdots$ Melchior [4] proved that

$$
t_{2} \geq 3+t_{4}+2 t_{5}+3 t_{6}+r t_{7}+\cdots .
$$

The following answers a question which the authors asked in [3].

Theorem 1. Absolute positive constants $C_{1}$ and $C_{2}$ exist such that the following is true: Let $0<\varepsilon \leq 1$. If $t_{3} \geq \varepsilon t_{2}$, then

$$
t \geq C_{1} \varepsilon n^{2}
$$

and

$$
t_{3} \geq C_{2} \varepsilon^{2} n^{2}
$$

We need the following:

Lemma 1. Let $n=r+s$ points be given, of which $r$ lie on a line $L$ and $s$ points do not. Then $t_{2} \geq r s-s(s-1)$. 
Proof. By induction on $s$.

Proof of Theorem 1. Suppose that $n$ points are given and that $t_{3} \geq \varepsilon t_{2}$. Let $L$ be the line containing the most points. If $|L|$ denotes the number of the $n$ points that are on $L$, then let $k=n-|L|$. Then no line contains more than $n-k$ points. It follows from Lemma 1 that $t_{2} \geq k(n-k)-k(k-1)$. We show that $t_{3} \leq \frac{1}{2} k(k-1)$ and $k>\frac{1}{2} n \varepsilon$. Let $t_{i}^{\prime}$ denote the number of lines containing exactly $i$ of the $k$ points not on $L$. To obtain the upper bound in $t_{3}$, note that there are only two types of lines containing exactly three of the $n$ points. There are at most $t_{2}^{\prime}$ lines containing one point of $L$ and at most $t_{3}^{\prime}$ lines containing no points of $L$. By counting pairs of the $k$ points, we see that

$$
t_{3} \leq t_{2}^{\prime}+t_{3}^{\prime} \leq t_{2}^{\prime}+3 t_{3}^{\prime} \leq \frac{1}{2} k(k-1)
$$

Hence,

$$
\frac{1}{2} k(k-1) \geq t_{3} \geq \varepsilon t_{2} \geq \varepsilon k(n-k)-\varepsilon k(k-1) .
$$

Dividing through by $\frac{1}{2} k$ gives $k-1 \geq 2 \varepsilon(n-3 k+1)$. From this, we may derive the lower bound on $k$. For, since $0<\varepsilon \leq 1$, we have

$$
4 k \geq(3 \varepsilon+1) k \geq 1+(2 n+1) \varepsilon>2 n \varepsilon,
$$

so that $k>\frac{1}{2} n \varepsilon$. However, at most $n-k$ points lie on any line, and so $t \geq C k n$ by a theorem of Szemerédi and Trotter [6] and Beck [1], where $C>0$. This gives us (2) with $C_{1}=\frac{1}{2} C$.

To obtain (3), we observe that

$$
(2+\varepsilon) t_{3}=2 t_{3}+\varepsilon t_{3} \geq 2 \varepsilon t_{2}+\varepsilon t_{3}=\varepsilon\left(2 t_{2}+t_{3}\right) .
$$

By adding $t_{2}+t_{3}$ to both sides of Melchior's inequality (1), we obtain a useful inequality due to Elliott [2]:

$$
2 t_{2}+t_{3} \geq 3+t_{2}+t_{3}+t_{4}+2 t_{5}+3 t_{6}+\cdots \geq 3+t .
$$

Hence

$$
3 t_{3} \geq(2+\varepsilon) t_{3} \geq \varepsilon(3+t)>\varepsilon t>C_{1} \varepsilon^{2} n^{2},
$$

or

$$
t_{3}>C_{2} \varepsilon^{2} n^{2}, \quad \text { where } C_{2}=\frac{1}{3} C_{1},
$$

and Theorem 1 is proved.

\section{The Second Problem}

Another problem which the authors introduced in [3] is the following: Given a set $S$ of $n$ points in the plane, not all collinear, let $f(S)$ denote the minimum of $|T|$ over all sets of points $T$, such that $T$ is disjoint from $S$ and every line containing two or more points of $S$ contains at least one point of $T$. We then define $f(n)$ to be the 
minimum of $f(S)$ taken over all noncollinear sets $S$ such that $|S|=n$. How large is $f(n)$ ? That $f(n)>C_{3} n$ for some $C_{3}>0$ follows from the so-called "weak Dirac conjecture," proved in [1] - see also [5] - with a very small constant $C_{3}$. As for an upper bound, an example with $n-2$ points collinear shows easily that $f(n) \leq n-1$, but Dean Hickerson has found a construction showing that $f(n) \leq n-2$ for all $n \geq 6$.

We end the discussion with Hickerson's construction: Let $k$ be a positive integer. Let $S$ consist of the points $(0, j)$ and $(1, j)$ for $j=0,1, \ldots, k$, together with the points $A\left(\frac{1}{2}, \frac{1}{2}(k-1)\right), B\left(\frac{1}{2}, \frac{1}{2} k\right)$, and $C\left(\frac{1}{2}, \frac{1}{2}(k+1)\right)$. If we label four of the points of $S$ as $D(0,0), E(1,0), F(0, k)$, and $G(1, k)$, then every line determined by $S$ has slope $0, \infty, \pm 1, \pm 2, \ldots, \pm(k-1)$, except the six lines $A F, A G, C D, C E, D B G$, and $E B F$.

Let $H$ be the intersection of $A F$ and $C D$, and let $I$ be the intersection of $C E$ and $D B G$. Finally let $J$ be the intersection of the lines $A G$ and $E B F$. The set $T$ consists of the points $H, I$, and $J$, together with the points at infinity determined by the aforementioned slopes $0, \infty, \pm 1, \pm 2, \ldots, \pm(k-1)$. Then $S$ and $T$ satisfy the desired conditions and $|S|=2 k+5$ and $|T|=2 k+3$, showing that $f(n) \leq n-2$ if $n$ is odd.

When $n$ is even, the construction needs to be modified. Delete $C$ from $S$ and replace the points $H$ and $I$ in $T$ by the point $K$, the intersection of $A F$ and $D B G$. The two constructions together show that $f(n) \leq n-2$ for $n \geq 6$.

\section{References}

1. J. Beck, On the lattice property of the plane and some problems of Dirac, Motzkin and Erdös in combinatorial geometry, Combinatorica 3 (1983), 281-297.

2. P. D. T. A. Elliott, On the number of circles determined by $n$ points. Acta Math. Acad. Sci. Hungar. 18 (1967), 181-188.

3. P. Erdős and G. Purdy, Some combinatorial problems in the plane, J. Combin. Theory Ser. A 25 (1978), 205-210.

4. E. Melchior, Uber Vielseite der projektiven Ebene, Deutsche Math. 5 (1940), 461-475.

5. G. Purdy, Some combinatorial problems in the plane, Ann. N.Y. Acad. Sci., 440 (1985), 65-68.

6. E. Szemerédi and W. T. Trotter, Extremal problems in discrete geometry, Combinatorica, 3 (1983), 381-392.

Received March 12, 1994. 